\title{
Plastic Bronchitis in Children with Asthma
}

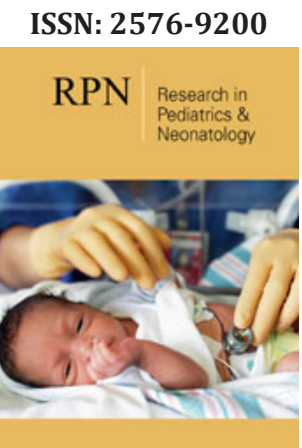

*Corresponding author: Pediatrics Department, Pediatric Infectious Diseases Division, Rabat children's hospital, Morocco

Submission: 想 February 20, 2020

Published: 眥 March 16, 2020

Volume 4 - Issue 3

How to cite this article: Rherib $\mathrm{C}$, Oudrhiri M, Benbrahim F, Benchekroun S, Mahraoui C, Hafidi NEL. Plastic Bronchitis in Children with Asthma. Res Pediatr Neonatol. 4(3).RPN.000588.2020. DOI: 10.31031/RPN.2020.04.000588

Copyright(C) Rherib C. This article is distributed under the terms of the Creative Commons Attribution 4.0 International License, which permits unrestricted use and redistribution provided that the original author and source are credited.

\author{
Rherib $\mathrm{C}^{1 *}$, Oudrhiri $\mathrm{M}^{1}$, Benbrahim $\mathrm{F}^{1}$, Benchekroun $\mathrm{S}^{1}$, Mahraoui $\mathrm{C}^{1}$ and \\ Hafidi NEL ${ }^{1,2}$ \\ ${ }^{1}$ Pediatrics Department, Morocco \\ ${ }^{2}$ Laboratory of Medical biotechnology, Morocco
}

\section{Abstract}

Plastic bronchitis (BP) is usually an evolutionary complication of respiratory conditions such as asthma and/ or pre-existing cardiac, and its occurrence in a patient without chronic respiratory pathology remains exceptional. We report the observation of a seven-year-old child, followed for asthma, who presented with a pneumatic appearance of commonplace, massive atelectasis of the entire left lung. Endoscopic exploration of the tracheobronchial tree confirmed the extensive obstruction of the left tree by bronchial mussels. The evolution was favorable after the extraction and spontaneous rejection of intrabronchial mussels.

Keywords: Plastic bronchitis; Bronchial mussels; Bronchoscopy; Asthma; Child

\section{Introduction}

Plastic bronchitis (PB) is a rare and frequently fatal condition characterized by expectoration of large, branching bronchial casts [1]. It has been described as a complication of respiratory diseases, including asthma, lymphatic abnormalities, infections and, in particular, congenital heart surgery [2]. We report a child's observation, followed for asthma, who presented with bronchitis plastic three years after the declaration of his asthma

\section{Case Report}

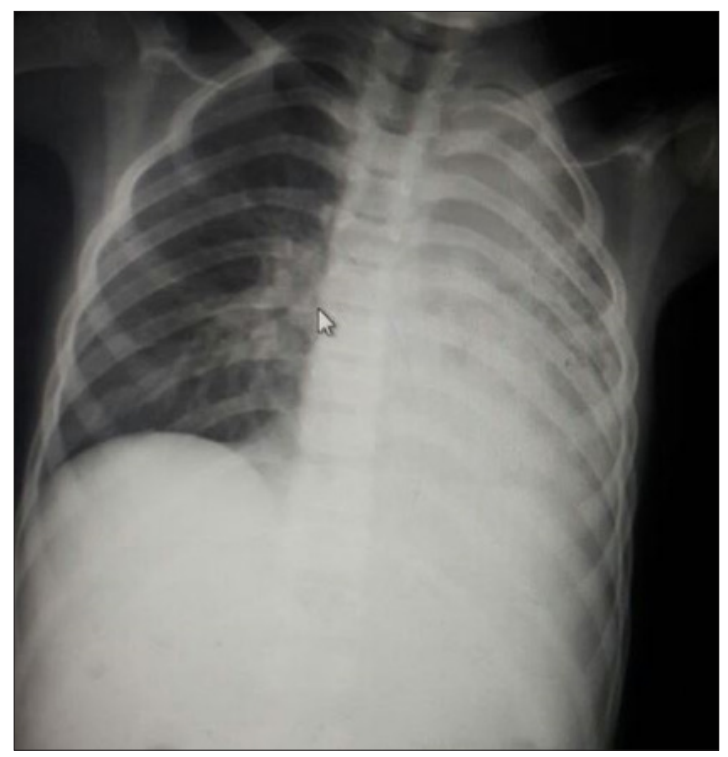

Figure 1: atelectasis of the left lung.

A seven-year-old boy, with a history of asthma under inhaled corticosteroid therapy, presented 20 days ago rhinorrhea associated with cough and fever at $39-40^{\circ} \mathrm{C}$, complicated by respiratory distress. On physical examination, he was feverish at $39{ }^{\circ} \mathrm{C}$, but hemodynamically stable; in moderate respiratory distress, tachypnic, with diminished breath sounds on the left. An anteroposterior chest radiograph revealed complete atelectasis of the left lung (Figure 1). Laboratory tests showed leukocytosis and high C-reactive protein (CRP); her blood cultures, cytobacteriological examination of sputum, her IDR and Koch's bacillus were negative. Pneumonia was suspected, so intravenous antibiotics were started, with respiratory 
physiotherapy without clinical or radiological improvement. Lungs Tomodensitometry revealed atelectasis of the upper and lower left lobes due to bronchial stenosis (Figure 2 \& 3). Bronchial fibroscopy was performed revealing the presence of a foreign body in the left main bronchus. Several fragments were extracted. The cytobacteriological study of fibroaspiration samples revealed the presence of Leiden thistle crystals with a negative culture; Histopathology demonstrated that the castings were composed of dense fibrinous debris with a mixed inflammatory rich in eosinophilic infiltrate. In view of this, that bronchitis plastic has been diagnosed and treated with macrolide, mycolitic and inhaled corticosteroid has been administered. The radiological évolution was progressively favorable with good aeration of the left lung (Figure 4) after extraction and spontaneous rejection bronchial mussels (Figure 5).

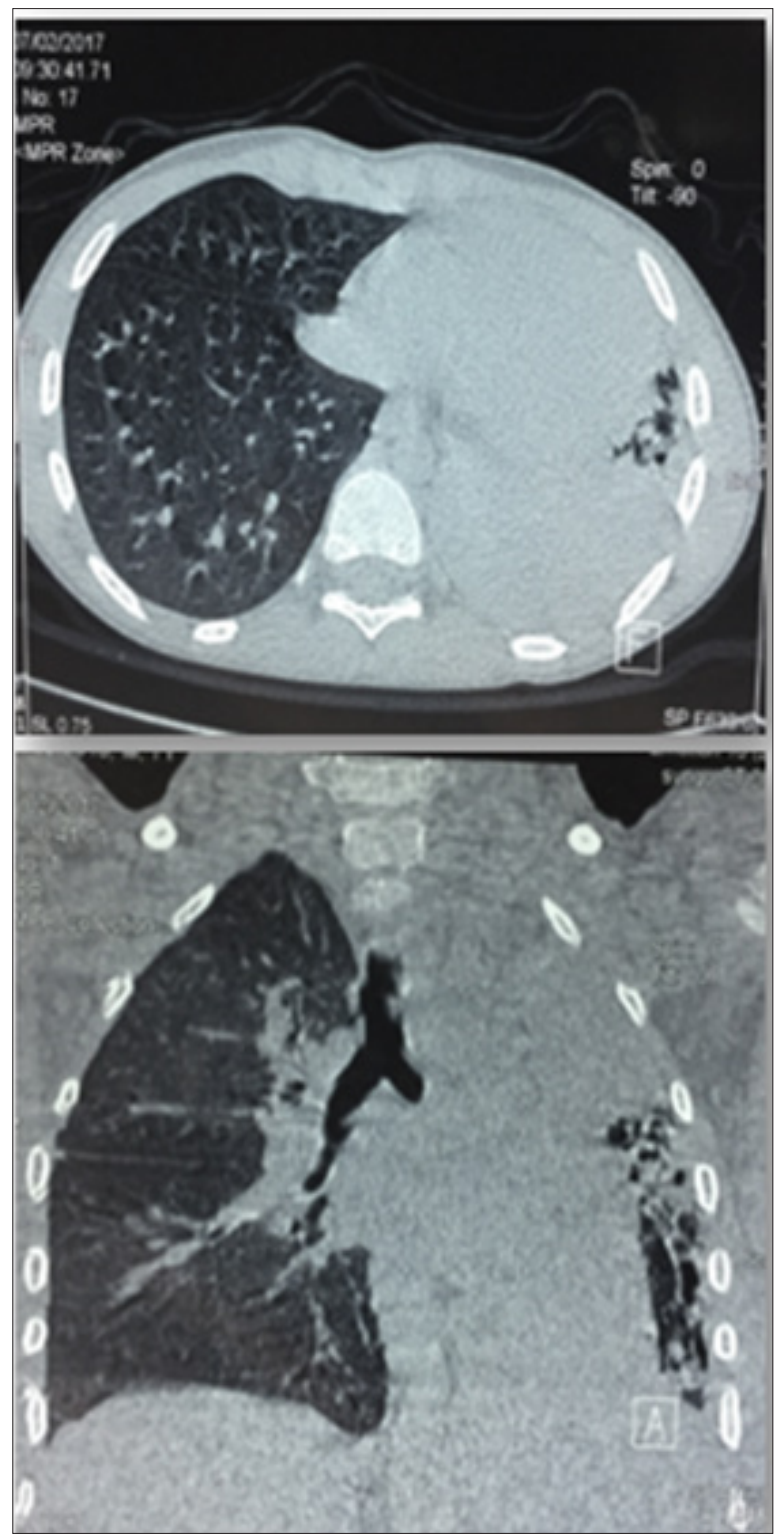

Figure 283: Thoracic computed tomography, total pulmonary collapse with mediastinal deviation.

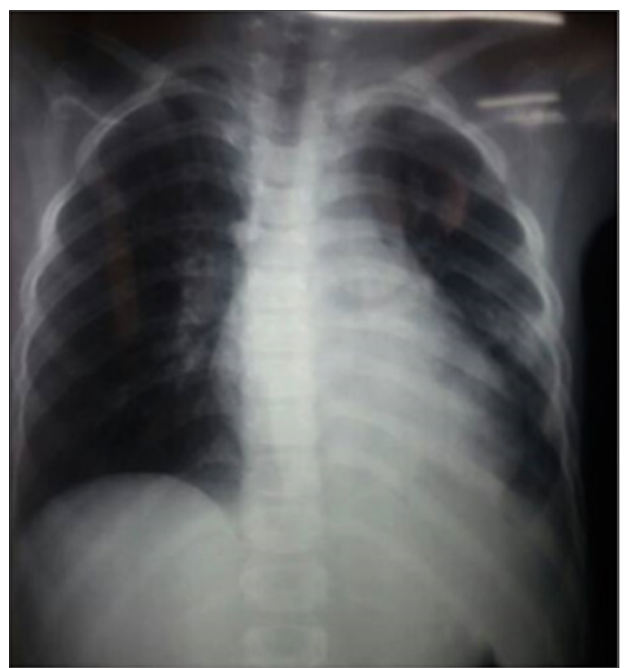

Figure 4: Chest X-ray after Endoscopic Extraction or Spontaneous Emission of Mussels: Reventilation of the Left Lung.

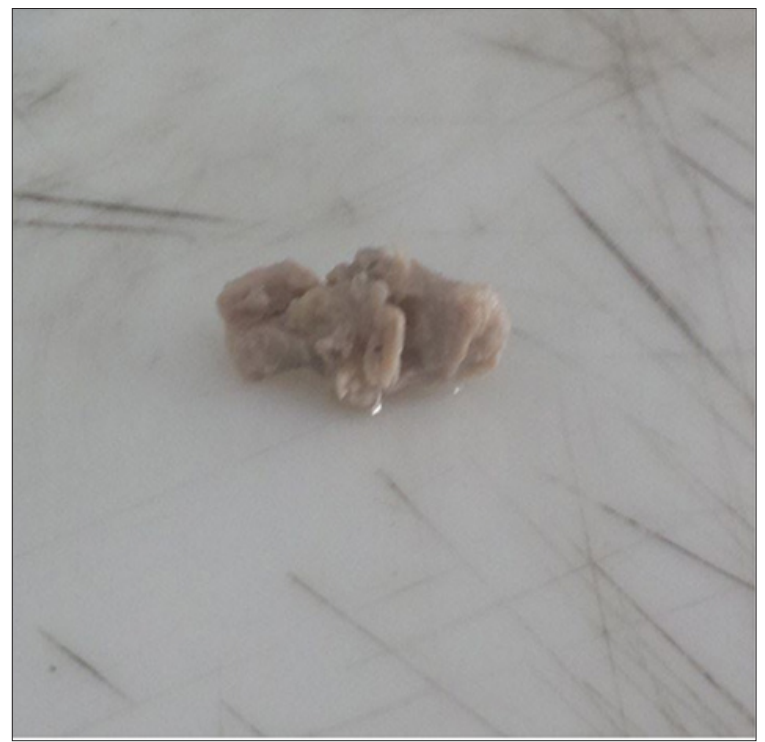

Figure 5: Intra-bronchial mold spontaneously rejected by the patient after coughing effort.

\section{Discussion}

The bronchitis plastic or disease of the bronchial mussels, is a rare affection defined by the extensive obstruction of the bronchial tree by thick molds, ramified and strongly adherent to the bronchial wall; they are rarely eliminated spontaneously in young children. All bronchial levels can be reached, especially the lower lobes; it affects both the child and the adult, but most cases have been reported in children. Its exact prevalence remains unknown and seems underestimated [3-5]. It is a rare cause of respiratory distress in children that usually complicates the development of pre-existing respiratory and/or cardiac conditions [3]. Plastic bronchitis has been called by different names over the years. It has been referred to as Hoffman's bronchitis, cast bronchitis, pseudomembranous bronchitis or fibrinous bronchitis [6] 
The most widely accepted classification is that of Sear, based on the characteristics of the bronchial cast and the underlying disease:

A. Type I (inflammatory) consists of fibrin casts, CharcotLeyden crystals and eosinophilia, and is generally associated with allergies or inflammatory diseases.

B. Type II consists of mucin casts and occurs with congenital heart disease [2] The clinical presentation has a productive cough, dyspnea, pleuritic chest pain, fever and wheezing.

Radiographic evaluation reveals the site of the bronchial cast impaction, demonstrating atelectasis or infiltrates. Hyperinflation is often evident on the contralateral side6. The CT scan allows visualization of impacted casts within the major airways. The diagnosis is usually confirmed by bronchoscopy, demonstrating airway obstruction from bronchial casts our patient is asthmatic [7]; however, Asthma and atopic disease are the next most common reported association with plastic bronchitis after congenital heart disease; According to Madsen $\mathrm{P}$ et al. [6] of the 22 cases reported with eosinophilic jets, 12 cases defined an atopic or asthmatic condition [6,7]. In addition to the endoscopic extraction of the mold, often essential in children, the treatment depends on the etiology. In patients with asthma or atopy and during cell projections the treatment must be directed to the underlying treatment inflammation. Inflammatory-type plastic bronchitis seems to be better controlled by inhaled or general corticotherapy than a cellular-type plastic bronchitis. Long-term oral antibiotic therapy with macrolides at low doses, by their modulating and mucoregulatory immune effect [3-5]. Bronchial mussels are sometimes recurrent. The preventive role of corticosteroid therapy in these recurrent forms is controversial [5].

\section{Conclusion}

Plastic bronchitis is a rare condition more and more known thanks to the development of endoscopic techniques pediatric. These must be proposed early to prevent the installation of bronchiectasis and limit the risk asphyxia in children.

\section{References}

1. Josipovic T, Kozak FK, Moxham JP, Chilvers M, Wensley D, et al. (2015) Casting a look at pediatric plastic bronchitis. Int J Pediatr Otorhinolaryngol 79(10): 1658-1661.

2. Abad PM, Pecellín ID, González Valencia JP (2015) Idiopathic plastic bronchitis as an uncommon cause of massive pulmonary atelectasis. Arch Bronconeumol 51(1): 46-47.

3. Khemiri M, Hammami O, Zouari S, Khaldi F, Barsaoui S (2008) Plastic bronchitis: About a pediatric observation plastic bronchitis: Report of a pediatric case. Journal of Clinical Pulmonology 64(5): 234-237.

4. Kouismi H, Zahraoui R, Bourkadi JE, Iraqi G (2013) La bronchite plastic: To suggest the observation of the plastic bronchitis: about one case. J Fran Viet Pneu 04(11): 1-44.

5. Amangar N, Moubachir H, Bourkadi JE, Barakat Z, Iraqi G (2013) Plastic bronchitis: About six pediatric cases plastic bronchitis: Report of six pediatric cases. French Journal of Allergology 53(8): 624-627.

6. Madsen P, Shah SA, Rubin BK (2005) Plastic bronchitis: New insights and a classification scheme. Paediatr Respir Rev 6(4): 292-300.

7. Kim EJ, Park JE, Kim DH, Lee J (2012) Plastic bronchitis in an adult with asthma. Tuberc Respir Dis (Seoul) 73(2): 122-126. 